hep-ph/9606481

KA-TP-13-1996

June 1996

\title{
Construction of a new constrained instanton in Yang-Mills-Higgs theory
}

\author{
F. R. Klinkhamer and J. Weller \\ Institut für Theoretische Physik \\ Universität Karlsruhe \\ D-76128 Karlsruhe
}

\begin{abstract}
A self-consistent ansatz is presented for a four-dimensional euclidean solution (instanton) in the vacuum sector of constrained $S U(2)$ Yang-Mills-Higgs theory.
\end{abstract}

PACS numbers : 11.15.Kc, 12.15.-y, 11.15.Bt, 12.38.Cy

keywords : classical solution, instanton, anomaly, summability

published in Nucl. Phys. B 481 (1996), 403 


\section{Introduction}

A topological argument has been given [1] for the existence of a new instanton ${ }^{\text {In }}$ in the vacuum sector of euclidean $S U(2)$ Yang-Mills-Higgs (YMH) theory, with a constraint added to fix the scale of the solution. The existence argument was based on the construction of a suitable non-contractible loop (NCL) of 4-dimensional field configurations. The basic idea now is that by making appropriate modifications of the configuration at the top of this particular NCL one arrives at an exact solution of the field equations. The present paper gives the resulting self-consistent ansatz for this new constrained instanton $\mathrm{I}^{\star}$. Technically the construction of $\mathrm{I}^{\star}$ is more complicated than for the related 3-dimensional sphaleron solution $\mathrm{S}^{\star} \llbracket$, but the method is essentially the same.

Most likely, $\mathrm{I}^{\star}$ is the lowest action constrained instanton solution in the vacuum sector of euclidean $S U(2)$ YMH theory. As such it is expected to be of fundamental importance for the quantum field theory of the electroweak interactions. In particular, $\mathrm{I}^{\star}$ is believed to play a role in the asymptotics of Feynman perturbation theory [5].

The outline of this paper is as follows. Section 2 describes briefly the theory considered. Section 3 presents the ansatz and Section 4 lists the symmetries, which are crucial for the derivation. Section 5 gives the action evaluated for the ansatz fields and Section 6 explains how the ansatz leads to having a non-trivial solution of the field equations. Section 7 , finally, gives a brief discussion of the potential physics applications mentioned above. There are also three appendices. Appendix A puts the NCL of [1] in a form appropriate for the construction of the ansatz of Section 3. Appendix B gives the transformations of the ansatz functions under the residual gauge symmetry discussed in Section 4. Appendix $\mathrm{C}$, finally, gives the ansatz actiondensity of Section 5 in a compact notation.

\section{Theory}

Consider a classical Yang-Mills theory, with non-Abelian gauge group $S U(2)$ and gauge coupling constant $g$, coupled to a Higgs scalar field in the fundamental representation, with vacuum expectation value $v$ and quartic coupling constant $\lambda$. The two mass scales of the theory are $M_{\mathrm{W}} \equiv \frac{1}{2} g v$ for the three $\mathrm{W}$ bosons and $M_{\mathrm{H}} \equiv \sqrt{2 \lambda} v$ for the single Higgs scalar. The total euclidean action is

$$
A=A_{\mathrm{YMH}}+A_{\mathrm{C}},
$$

*Here, and in the following, the term "instanton" refers to any localized, finite action solution of the general field equations belonging to the euclidean action of the theory considered, not exclusively to solutions of certain first order self-duality conditions [2, 3]. 
with the Yang-Mills-Higgs action

$$
A_{\mathrm{YMH}}=\int_{\mathbb{R}^{4}} d^{4} x\left[-\frac{1}{2 g^{2}} \operatorname{Tr} W_{\mu \nu}^{2}+\left|D_{\mu} \Phi\right|^{2}+\lambda\left(|\Phi|^{2}-\frac{v^{2}}{2}\right)^{2}\right],
$$

where $W_{\mu \nu} \equiv \partial_{\mu} W_{\nu}-\partial_{\nu} W_{\mu}+\left[W_{\mu}, W_{\nu}\right], D_{\mu} \Phi \equiv\left(\partial_{\mu}+W_{\mu}\right) \Phi, W_{\mu} \equiv W_{\mu}^{a} \sigma^{a} /(2 i)$ and $\sigma^{a}$ are the standard Pauli matrices, and a constraint term [6]

$$
A_{\mathrm{C}}=\frac{\kappa}{g^{2}}\left(\frac{1}{M_{W}^{4}} \int_{\mathbb{R}^{4}} d^{4} x O_{8}-\frac{8 \pi^{2} c}{\left(M_{\mathrm{W}} \varrho\right)^{4}}\right),
$$

with $\kappa$ an arbitrary positive constant (Lagrange multiplier) and $\varrho$ the "size" of the field configuration. Specifically, we choose for the constraint operator

$$
O_{8}=q_{\mathrm{P}}^{2}
$$

where $q_{\mathrm{P}}$ is the Pontryagin density

$$
q_{\mathrm{P}} \equiv-\frac{1}{4} \epsilon_{\kappa \lambda \mu \nu} \operatorname{Tr} W_{\kappa \lambda} W_{\mu \nu},
$$

and for the numerical constant $c$ we take the value

$$
c=\frac{288}{21} \text {. }
$$

This particular choice for $c$ reproduces the usual scale parameter of a BPST [2] instantonanti-instanton pair at infinite separation. Of course, there are many other constraint operators $O_{d}$ possible, as long as they have canonical mass dimension $d>4$. Also, there might, in principle, exist solutions for which the single constraint operator (4) does not suffice and further terms need to be added.

Having defined the theory we proceed in three steps. First, we obtain for a given positive value of $\kappa$ a solution $W^{\star}, \Phi^{\star}$ of the field equations resulting from variations $\delta W, \delta \Phi$ of the total action $A$. Second, we solve for the scale $\varrho^{\star}=\varrho^{\star}\left(\kappa, W^{\star}, \Phi^{\star}\right)$, so that $A_{\mathrm{C}}^{\star}=0$ and $A^{\star}=A_{\mathrm{YMH}}^{\star}\left(\kappa, W^{\star}, \Phi^{\star}\right)$, and eliminate $\kappa$ between $\varrho^{\star}$ and $A_{\mathrm{YMH}}^{\star}$. Third, with these values $\varrho^{\star}$ and $A_{\mathrm{YMH}}^{\star}\left(\varrho^{\star}, W^{\star}, \Phi^{\star}\right)$, we integrate over the collective coordinate $\varrho$ in the path integral of the particular Green's function considered. Further details may be found in [1], [].

Here, we focus on the first crucial step, namely to discover a non-trivial solution of the field equations belonging to the euclidean action (11). More specificially, we look for a finite action solution with Pontryagin index (topological charge)

$$
Q_{\mathrm{P}} \equiv \frac{1}{8 \pi^{2}} \int_{\mathbb{R}^{4}} d^{4} x q_{\mathrm{P}}
$$

vanishing, i. e. a new constrained instanton in the vacuum sector. 


\section{Ansatz}

Define cylindrical coordinates $\rho, \varphi, z$ and $\tau$ in terms of the cartesian coordinates

$$
\left(x_{1}, x_{2}, x_{3}, x_{4}\right) \equiv(\rho \cos \varphi, \rho \sin \varphi, z, \tau)
$$

and introduce a triad of matrices

$$
\begin{aligned}
\mathbf{u} & \equiv \sin \varphi \tau^{2}+\cos \varphi \tau^{1} \\
\mathbf{v} & \equiv \cos \varphi \tau^{2}-\sin \varphi \tau^{1} \\
\mathbf{w} & \equiv \tau^{3}
\end{aligned}
$$

with $\tau^{a} \equiv \sigma^{a} /(2 i)$ and $\sigma^{a}$ the Pauli matrices

$$
\sigma^{1} \equiv\left(\begin{array}{cc}
0 & 1 \\
1 & 0
\end{array}\right) \quad \sigma^{2} \equiv\left(\begin{array}{cc}
0 & -i \\
i & 0
\end{array}\right) \quad \sigma^{3} \equiv\left(\begin{array}{cc}
1 & 0 \\
0 & -1
\end{array}\right) .
$$

The ansatz is then given by

$$
\begin{aligned}
W_{1} & =\frac{1}{\rho}\left\{\left(C_{1} \cos \varphi-C_{3} \sin \varphi\right) \mathbf{u}+\left(C_{2} \cos \varphi-C_{4} \sin \varphi\right) \mathbf{v}+\left(C_{5} \sin \varphi+C_{6} \cos \varphi\right) \mathbf{w}\right\} \\
W_{2} & =\frac{1}{\rho}\left\{\left(C_{1} \sin \varphi+C_{3} \cos \varphi\right) \mathbf{u}+\left(C_{2} \sin \varphi+C_{4} \cos \varphi\right) \mathbf{v}-\left(C_{5} \cos \varphi-C_{6} \sin \varphi\right) \mathbf{w}\right\} \\
W_{3} & =\frac{1}{z}\left\{C_{7} \mathbf{u}-C_{8} \mathbf{v}+C_{9} \mathbf{w}\right\} \\
W_{4} & =\frac{1}{\tau}\left\{C_{10} \mathbf{u}-C_{11} \mathbf{v}+C_{12} \mathbf{w}\right\} \\
\Phi & =\frac{v}{\sqrt{2}}\left(\begin{array}{c}
-\left(H_{2}+i H_{1}\right) e^{-i \varphi} \\
H_{0}+i H_{3}
\end{array}\right),
\end{aligned}
$$

with

$$
\begin{gathered}
C_{1}=4 \frac{\rho}{X} f_{1} \quad C_{2}=4 \frac{\rho z}{X^{2}} f_{2} \quad C_{3}=4 \frac{\rho z}{X^{2}} f_{3} \quad C_{4}=4 \frac{\rho}{X} f_{4} \\
C_{5}=4 \frac{\rho^{2}}{X^{2}} f_{5} \quad C_{6}=4 \frac{\rho^{2} z}{X^{3}} f_{6} \\
C_{7}=4 \frac{\rho z^{2}}{X^{3}} f_{7} \quad C_{8}=4 \frac{\rho z}{X^{2}} f_{8} \quad C_{9}=4 \frac{z}{X} f_{9} \\
C_{10}=4 \frac{\rho \tau}{X^{2}} f_{10} \quad C_{11}=4 \frac{\rho z \tau}{X^{3}} f_{11} \quad C_{12}=4 \frac{\tau z}{X^{2}} f_{12} \\
H_{0}=\frac{z}{X} h_{0} \quad H_{1}=2 \frac{\rho z}{X^{2}} h_{1} \quad H_{2}=2 \frac{\rho}{X} h_{2} \quad H_{3}=h_{3}
\end{gathered}
$$

and

$$
X^{2} \equiv x^{2}+x_{a}^{2} \equiv \rho^{2}+z^{2}+\tau^{2}+x_{a}^{2}
$$


for arbitrary parameter $x_{a}$. The axial functions $f_{i}=f_{i}(\rho, z, \tau)$ and $h_{j}=h_{j}(\rho, z, \tau)$, with $i=1, \ldots, 12$ and $j=0, \ldots, 3$, are non-singular and have reflection symmetry

$$
\begin{aligned}
f_{i}(\rho,-z, \tau) & =f_{i}(\rho, z, \tau) \\
h_{j}(\rho,-z, \tau) & =h_{j}(\rho, z, \tau) .
\end{aligned}
$$

Continuity of the gauge fields at $\rho=0$ demands

$$
\begin{aligned}
f_{1}(0, z, \tau) & =f_{4}(0, z, \tau) \\
-f_{2}(0, z, \tau) & =f_{3}(0, z, \tau) .
\end{aligned}
$$

All axial functions have furthermore Neumann boundary conditions at $\rho=0$

$$
\begin{array}{r}
\partial_{\rho} f_{i}(0, z, \tau)=0 \\
\partial_{\rho} h_{j}(0, z, \tau)=0
\end{array}
$$

and Dirichlet-like boundary conditions at infinity

$$
\lim _{|x| \rightarrow \infty}\left[\begin{array}{c}
f_{1} \\
f_{2} \\
f_{3} \\
f_{4} \\
f_{5} \\
f_{6} \\
f_{7} \\
f_{8} \\
f_{9} \\
f_{10} \\
f_{11} \\
f_{12} \\
h_{0} \\
h_{1} \\
h_{2} \\
h_{3}
\end{array}\right]=\left[\begin{array}{c}
\tau / x \\
-1 \\
\left(z^{2}-\rho^{2}+\tau^{2}\right) / x^{2} \\
\tau\left(z^{2}-\rho^{2}+\tau^{2}\right) / x^{3} \\
2\left(\tau^{2}+z^{2}\right) / x^{2} \\
0 \\
-2 \tau / x \\
\left(\tau^{2}-\rho^{2}-z^{2}\right) / x^{2} \\
2 \tau \rho^{2} / x^{3} \\
\left(z^{2}-\rho^{2}-\tau^{2}\right) / x^{2} \\
-2 \tau / x \\
-2 \rho^{2} / x^{2} \\
0 \\
-1 \\
-\tau / x \\
\left(\rho^{2}-z^{2}-\tau^{2}\right) / x^{2}
\end{array}\right] .
$$

The ansatz (8) has, as will be explained in the next Section, a residual gauge symmetry, which allows for the elimination of essentially three functions. One possible gauge choice is the well-known radial gauge $\hat{x}_{\mu} W_{\mu}=0$ :

$$
C_{1}+C_{7}+C_{10}=C_{2}-C_{8}-C_{11}=C_{6}+C_{9}+C_{12}=0 .
$$

This completes the description of the ansatzf. Next, we study its symmetries and explain the logic behind the construction.

*The $U(1)$ hypercharge gauge field of the electroweak standard model can easily be included, just as for the sphaleron $\mathrm{S}^{\star}$ 顿. 


\section{Symmetries}

The ansatz of the previous Section has, by construction, several symmetries, which we will now discuss.

\subsection{Axial symmetry}

Generally, scalar and vector fields transform under infinitesimal coordinate transformations

$$
x^{\mu} \rightarrow x^{\mu}+\delta \alpha \xi^{\mu}(x)
$$

as follows:

$$
\begin{aligned}
\Phi(x) & \rightarrow \Phi(x)+\delta \alpha \mathbb{L}_{\xi} \Phi(x) \\
W_{\mu}(x) & \rightarrow W_{\mu}(x)+\delta \alpha \mathbb{L}_{\xi} W_{\mu}(x)
\end{aligned}
$$

with Lie derivatives

$$
\begin{aligned}
\mathbb{L}_{\xi} \Phi & \equiv \xi^{\nu} \partial_{\nu} \Phi \\
\mathbb{L}_{\xi} W_{\mu} & \equiv \xi^{\nu} \partial_{\nu} W_{\mu}+\left(\partial_{\mu} \xi^{\nu}\right) W_{\nu}
\end{aligned}
$$

In the present paper we will use a generalized form of invariance of the fields, namely by allowing for a compensating internal symmetry transformation. In short, invariance need only hold up to a gauge transformation (or any other internal symmetry transformation for that matter).

The coordinate transformation relevant here is the rotation

$$
x^{\mu} \rightarrow x^{\mu}+\delta \varphi \xi_{\mathrm{R}}^{\mu},
$$

generated by the vector field

$$
\xi_{\mathrm{R}}^{\mu}=\left(-x_{2}, x_{1}, 0,0\right)
$$

and infinitesimal parameter $\delta \varphi$. It is then straightforward to verify the invariance of the ansatz gauge fields (8) under the transformation (16b), with $\delta \alpha$ replaced by $\delta \varphi$ and $\xi$ by $\xi_{\mathrm{R}}$, combined with the global gauge transformation

$$
W_{\mu} \rightarrow W_{\mu}-\left[W_{\mu}, \tau^{3}\right] \delta \varphi
$$

For the scalar field (8) there is in addition to the transformation (16a), again with $\delta \alpha$ replaced by $\delta \varphi$ and $\xi$ by $\xi_{\mathrm{R}}$, a compensating transformation

$$
\Phi \rightarrow \Phi-i \delta \varphi\left(\frac{1+\sigma^{3}}{2}\right) \Phi,
$$

which combines a global $S U(2)$ gauge transformation with a $U(1)$ phase transformation. The ansatz (8) thus has $U(1)$ invariance under rotations in the $x_{1}, x_{2}$ plane. 


\section{$4.2 \quad$ Discrete symmetries}

The ansatz (8 10) is invariant under the combined discrete transformation

$$
P_{x z} \otimes C \otimes G_{\mathrm{c}},
$$

with $P_{x z}$ the following parity-like transformation of the classical fields (cartesian coordinates $x_{1}, x_{2}, x_{3}, x_{4}$ being written as $\left.x, y, z, \tau\right)$ :

$$
\left[\begin{array}{c}
W_{1} \\
W_{2} \\
W_{3} \\
W_{4} \\
\Phi
\end{array}\right](x, y, z, \tau) \rightarrow\left[\begin{array}{r}
-W_{1} \\
W_{2} \\
-W_{3} \\
W_{4} \\
\Phi
\end{array}\right](-x, y,-z, \tau)
$$

$C$ the charge conjugation transformation

$$
\left[\begin{array}{c}
W_{\mu} \\
\Phi
\end{array}\right] \rightarrow\left[\begin{array}{c}
W_{\mu}^{*} \\
\Phi^{*}
\end{array}\right]
$$

and $G_{\mathrm{c}}$ the global $S U(2)$ gauge transformation with gauge parameter function $\Gamma=-\mathbb{1}_{2}$ in the center of the group

$$
\left[\begin{array}{c}
W_{\mu} \\
\Phi
\end{array}\right] \rightarrow\left[\begin{array}{c}
W_{\mu} \\
-\Phi
\end{array}\right]
$$

The second discrete symmetry of the ansatz acts only on the fields in the $z=0$ plane and corresponds to the usual parity transformation $P$

$$
\left[\begin{array}{c}
W_{1} \\
W_{2} \\
W_{4} \\
\Phi
\end{array}\right](x, y, 0, \tau) \rightarrow\left[\begin{array}{c}
-W_{1} \\
-W_{2} \\
-W_{4} \\
\Phi
\end{array}\right](-x,-y, 0,-\tau)
$$

provided the ansatz functions (9) obey the following conditions :

$$
\begin{aligned}
-f_{1}(\rho, 0,-\tau) & =f_{1}(\rho, 0, \tau) \\
-f_{4}(\rho, 0,-\tau) & =f_{4}(\rho, 0, \tau) \\
f_{5}(\rho, 0,-\tau) & =f_{5}(\rho, 0, \tau) \\
f_{10}(\rho, 0,-\tau) & =f_{10}(\rho, 0, \tau) \\
-h_{2}(\rho, 0,-\tau) & =h_{2}(\rho, 0, \tau) \\
h_{3}(\rho, 0,-\tau) & =h_{3}(\rho, 0, \tau) .
\end{aligned}
$$

Note that the same parity reflection symmetry holds for the sphaleron $S^{\star}$ 四, which corresponds in fact to the $z=0$ slice of $\mathrm{I}^{\star}$. 
Both discrete symmetries also distinguish the configuration at the "top" of the NCL constructed previously [1]. The crucial idea behind the ansatz of Section 3 was to generalize that particular NCL configuration, while respecting the axial and discrete symmetries present. Further details can be found in Appendix A.

\subsection{Residual gauge symmetry}

The axisymmetric ansatz (8) is form invariant under gauge transformations

$$
\begin{aligned}
W_{\mu} & \rightarrow \Gamma\left(W_{\mu}+\partial_{\mu}\right) \Gamma^{-1} \\
\Phi & \rightarrow \Gamma \Phi
\end{aligned}
$$

with gauge parameter function

$$
\Gamma=\exp \left(\omega_{1} \mathbf{u}+\omega_{2} \mathbf{v}+\omega_{3} \mathbf{w}\right)
$$

where $\omega_{a}=\omega_{a}(\rho, z, \tau)$. In order to maintain the discrete symmetries of the ansatz, there are the following conditions :

$$
\begin{aligned}
\omega_{1}(\rho,-z, \tau) & =\omega_{1}(\rho, z, \tau) \\
-\omega_{2}(\rho,-z, \tau) & =\omega_{2}(\rho, z, \tau) \\
-\omega_{3}(\rho,-z, \tau) & =\omega_{3}(\rho, z, \tau) \\
-\omega_{1}(\rho, 0,-\tau) & =\omega_{1}(\rho, 0, \tau) .
\end{aligned}
$$

The explicit transformations of the coefficient functions $C_{i}$ and $H_{j}$ are somewhat involved and are given in Appendix B. As mentioned above, these residual gauge transformations eliminate essentially 3 functions from the ansatz.

\section{Action}

The ansatz of section 3 gives for the Yang-Mills-Higgs action

$$
A_{\mathrm{YMH}}=4 \pi \int_{-\infty}^{\infty} d \tau \int_{0}^{\infty} d z \int_{0}^{\infty} d \rho \rho a_{\mathrm{YMH}}
$$

with actiondensity

$$
a_{\mathrm{YMH}}=a_{\mathrm{WKIN}}+a_{\mathrm{HKIN}}+a_{\mathrm{HPOT}},
$$


where

$$
\begin{aligned}
& a_{\mathrm{WKIN}}= \\
& \frac{1}{2 g^{2}}\left\{\left(\frac{\partial_{z} C_{1}}{\rho}-\frac{\partial_{\rho} C_{7}}{z}-\frac{C_{2} C_{9}}{\rho z}-\frac{C_{6} C_{8}}{\rho z}\right)^{2}+\left(\frac{\partial_{\tau} C_{1}}{\rho}-\frac{\partial_{\rho} C_{10}}{\tau}-\frac{C_{2} C_{12}}{\rho \tau}-\frac{C_{6} C_{11}}{\rho \tau}\right)^{2}\right. \\
& +\left(\frac{\partial_{z} C_{2}}{\rho}+\frac{\partial_{\rho} C_{8}}{z}+\frac{C_{1} C_{9}}{\rho z}-\frac{C_{6} C_{7}}{\rho z}\right)^{2}+\left(\frac{\partial_{\tau} C_{2}}{\rho}+\frac{\partial_{\rho} C_{11}}{\tau}+\frac{C_{1} C_{12}}{\rho \tau}-\frac{C_{6} C_{10}}{\rho \tau}\right)^{2} \\
& +\left(\frac{\partial_{z} C_{6}}{\rho}-\frac{\partial_{\rho} C_{9}}{z}+\frac{C_{1} C_{8}}{\rho z}+\frac{C_{2} C_{7}}{\rho z}\right)^{2}+\left(\frac{\partial_{\tau} C_{6}}{\rho}-\frac{\partial_{\rho} C_{12}}{\tau}+\frac{C_{1} C_{11}}{\rho \tau}+\frac{C_{2} C_{10}}{\rho \tau}\right)^{2} \\
& +\left(\frac{\partial_{\tau} C_{7}}{z}-\frac{\partial_{z} C_{10}}{\tau}+\frac{C_{8} C_{12}}{z \tau}-\frac{C_{9} C_{11}}{z \tau}\right)^{2}+\left(\frac{\partial_{\tau} C_{8}}{z}-\frac{\partial_{z} C_{11}}{\tau}-\frac{C_{7} C_{12}}{z \tau}+\frac{C_{9} C_{10}}{z \tau}\right)^{2} \\
& +\left(\frac{\partial_{\tau} C_{9}}{z}-\frac{\partial_{z} C_{12}}{\tau}+\frac{C_{7} C_{11}}{z \tau}-\frac{C_{8} C_{10}}{z \tau}\right)^{2}+\left(\frac{\partial_{\rho} C_{3}}{\rho}-\frac{C_{4} C_{6}}{\rho^{2}}-\frac{C_{2}}{\rho^{2}}\left(C_{5}-1\right)\right)^{2} \\
& +\left(\frac{\partial_{z} C_{3}}{\rho}-\frac{C_{4} C_{9}}{\rho z}+\frac{C_{8}}{\rho z}\left(C_{5}-1\right)\right)^{2}+\left(\frac{\partial_{\tau} C_{3}}{\rho}-\frac{C_{4} C_{12}}{\rho \tau}+\frac{C_{11}}{\rho \tau}\left(C_{5}-1\right)\right)^{2} \\
& +\left(\frac{\partial_{\rho} C_{4}}{\rho}+\frac{C_{3} C_{6}}{\rho^{2}}+\frac{C_{1}}{\rho^{2}}\left(C_{5}-1\right)\right)^{2}+\left(\frac{\partial_{z} C_{4}}{\rho}+\frac{C_{3} C_{9}}{\rho z}+\frac{C_{7}}{\rho z}\left(C_{5}-1\right)\right)^{2} \\
& +\left(\frac{\partial_{\tau} C_{4}}{\rho}+\frac{C_{3} C_{12}}{\rho \tau}+\frac{C_{10}}{\rho \tau}\left(C_{5}-1\right)\right)^{2}+\left(\frac{\partial_{\rho} C_{5}}{\rho}+\frac{C_{2} C_{3}}{\rho^{2}}-\frac{C_{1} C_{4}}{\rho^{2}}\right)^{2} \\
& \left.+\left(\frac{\partial_{z} C_{5}}{\rho}-\frac{C_{3} C_{8}}{\rho z}-\frac{C_{4} C_{7}}{\rho z}\right)^{2}+\left(\frac{\partial_{\tau} C_{5}}{\rho}-\frac{C_{3} C_{11}}{\rho \tau}-\frac{C_{4} C_{10}}{\rho \tau}\right)^{2}\right\}
\end{aligned}
$$

$$
\begin{aligned}
& a_{\mathrm{HKIN}}= \\
& \frac{v^{2}}{2}\left\{\left(\partial_{\rho} H_{0}-\frac{H_{1} C_{1}}{2 \rho}-\frac{H_{2} C_{2}}{2 \rho}-\frac{H_{3} C_{6}}{2 \rho}\right)^{2}+\left(\partial_{z} H_{0}-\frac{H_{1} C_{7}}{2 z}+\frac{H_{2} C_{8}}{2 z}-\frac{H_{3} C_{9}}{2 z}\right)^{2}\right. \\
&+\quad\left(\partial_{\tau} H_{0}-\frac{H_{1} C_{10}}{2 \tau}+\frac{H_{2} C_{11}}{2 \tau}-\frac{H_{3} C_{12}}{2 \tau}\right)^{2}+\left(\partial_{\rho} H_{1}+\frac{H_{0} C_{1}}{2 \rho}-\frac{H_{2} C_{6}}{2 \rho}+\frac{H_{3} C_{2}}{2 \rho}\right)^{2} \\
&+\quad\left(\partial_{z} H_{1}+\frac{H_{0} C_{7}}{2 z}-\frac{H_{2} C_{9}}{2 z}-\frac{H_{3} C_{8}}{2 z}\right)^{2}+\left(\partial_{\tau} H_{1}+\frac{H_{0} C_{10}}{2 \tau}-\frac{H_{2} C_{12}}{2 \tau}-\frac{H_{3} C_{11}}{2 \tau}\right)^{2} \\
&+\quad\left(\partial_{\rho} H_{2}+\frac{H_{0} C_{2}}{2 \rho}+\frac{H_{1} C_{6}}{2 \rho}-\frac{H_{3} C_{1}}{2 \rho}\right)^{2}+\left(\partial_{z} H_{2}-\frac{H_{0} C_{8}}{2 z}+\frac{H_{1} C_{9}}{2 z}-\frac{H_{3} C_{7}}{2 z}\right)^{2} \\
&+\quad\left(\partial_{\tau} H_{2}-\frac{H_{0} C_{11}}{2 \tau}+\frac{H_{1} C_{12}}{2 \tau}-\frac{H_{3} C_{10}}{2 \tau}\right)^{2}+\left(\partial_{\rho} H_{3}+\frac{H_{0} C_{6}}{2 \rho}-\frac{H_{1} C_{2}}{2 \rho}+\frac{H_{2} C_{1}}{2 \rho}\right)^{2}
\end{aligned}
$$




$$
\begin{aligned}
+ & \left(\partial_{z} H_{3}+\frac{H_{0} C_{9}}{2 z}+\frac{H_{1} C_{8}}{2 z}+\frac{H_{2} C_{7}}{2 z}\right)^{2}+\left(\partial_{\tau} H_{3}+\frac{H_{0} C_{12}}{2 \tau}+\frac{H_{1} C_{11}}{2 \tau}+\frac{H_{2} C_{10}}{2 \tau}\right)^{2} \\
+ & \left(\frac{H_{0} C_{4}}{2 \rho}+\frac{H_{1}}{\rho}-\frac{H_{1} C_{5}}{2 \rho}-\frac{H_{3} C_{3}}{2 \rho}\right)^{2}+\left(\frac{H_{0} C_{3}}{2 \rho}-\frac{H_{2}}{\rho}+\frac{H_{2} C_{5}}{2 \rho}+\frac{H_{3} C_{4}}{2 \rho}\right)^{2} \\
+ & \left.\left(\frac{H_{0} C_{5}}{2 \rho}+\frac{H_{1} C_{4}}{2 \rho}-\frac{H_{2} C_{3}}{2 \rho}\right)^{2}+\left(\frac{H_{1} C_{3}}{2 \rho}+\frac{H_{2} C_{4}}{2 \rho}-\frac{H_{3} C_{5}}{2 \rho}\right)^{2}\right\} \\
a_{\text {нРот }} & =\lambda \frac{v^{4}}{4}\left(H_{0}^{2}+H_{1}^{2}+H_{2}^{2}+H_{3}^{2}-1\right)^{2} .
\end{aligned}
$$

For brevity, we have not made the functions $C_{i}$ and $H_{j}$ explicit by inserting (9), but if one does one readily verifies that the action density is finite everywhere and vanishes at infinity. In addition, we can fix the gauge with conditions (14).

The constraint operator (3, 4) gives for the ansatz fields (8) a term in the total action of the form

$$
A_{\mathrm{C}}=\frac{\kappa}{g^{2}}\left(\frac{4 \pi}{M_{W}^{4}} \int_{-\infty}^{\infty} d \tau \int_{0}^{\infty} d z \int_{0}^{\infty} d \rho \rho q_{\mathrm{P}}^{2}-\frac{8 \pi^{2} c}{\left(M_{\mathrm{W}} \varrho\right)^{4}}\right),
$$

with Pontryagin density $q_{\mathrm{P}}$ given by

$$
\begin{aligned}
\rho^{2} z \tau q_{\mathrm{P}} & =C_{1} \stackrel{\leftrightarrow}{D}_{\tau z} C_{3}+C_{2} \stackrel{\leftrightarrow}{D}_{\tau z} C_{4}+C_{5} \stackrel{\leftrightarrow}{D}_{\tau z} C_{6}+C_{7} \stackrel{\leftrightarrow}{D}_{\rho \tau} C_{3} \\
& +C_{4} \stackrel{\leftrightarrow}{D}_{\rho \tau} C_{8}+C_{5} \stackrel{\leftrightarrow}{D}_{\rho \tau} C_{9}+C_{3} \stackrel{\leftrightarrow}{D}_{\rho z} C_{10}+C_{11} \stackrel{\leftrightarrow}{D}_{\rho z} C_{4}+C_{12} \stackrel{\leftrightarrow}{D}_{\rho z} C_{5} \\
& +\rho \vec{\partial}_{\rho}\left[\left(C_{5}-1\right)\left(C_{7} C_{11}-C_{8} C_{10}\right)+C_{9}\left(C_{3} C_{11}+C_{4} C_{10}\right)-C_{12}\left(C_{3} C_{8}+C_{4} C_{7}\right)\right] \\
& +z \vec{\partial}_{z}\left[\left(1-C_{5}\right)\left(C_{2} C_{10}+C_{1} C_{11}\right)-C_{6}\left(C_{3} C_{11}+C_{4} C_{10}\right)+C_{12}\left(C_{1} C_{4}-C_{2} C_{3}\right)\right] \\
& +\tau \vec{\partial}_{\tau}\left[\left(C_{5}-1\right)\left(C_{1} C_{8}+C_{2} C_{7}\right)+C_{6}\left(C_{3} C_{8}+C_{4} C_{7}\right)-C_{9}\left(C_{1} C_{4}-C_{2} C_{3}\right)\right]
\end{aligned}
$$

in terms of

$$
\stackrel{\leftrightarrow}{D}_{\tau z} \equiv \overleftarrow{\partial}_{\tau} \tau z \vec{\partial}_{z}-\overleftarrow{\partial}_{z} z \tau \vec{\partial}_{\tau}
$$

and similarly for $\stackrel{\leftrightarrow}{D}_{\rho \tau}$ and $\stackrel{\leftrightarrow}{D}_{\rho z}$, where the partial derivatives $\overleftarrow{\partial}$ and $\vec{\partial}$ operate to the left and to the right, respectively. Again, we have to insert (9) and implement the gauge fixing conditions (14).

It is possible to get more compact expressions for the Pontryagin density and actiondensities, see Appendix C. With (31, 36) one then obtains the final expression for the total action (1- 6) evaluated with the ansatz fields. Furthermore, the Pontryaginindex (7) 
can be shown to vanish for the fields of the ansatz. This can be established most easily by use of the Chern-Simons current

$$
j_{\mu} \equiv-\epsilon_{\mu \nu \alpha \beta} \operatorname{Tr}\left[W_{\nu}\left(\partial_{\alpha} W_{\beta}+\frac{2}{3} W_{\alpha} W_{\beta}\right)\right],
$$

whose divergence gives the Pontryagin density $\partial_{\mu} j_{\mu}=q_{\mathrm{P}}$, so that

$$
Q_{\mathrm{P}}=\frac{1}{8 \pi^{2}} \oint_{S_{\infty}^{3}} d \Sigma_{\mu} j_{\mu} .
$$

For the ansatz fields at infinity $(8,9,13)$ the Chern-Simons current vanishes identically and

$$
Q_{\mathrm{P}}\left(W_{\mathrm{I}^{\star}}\right)=0 .
$$

This result for the topological charge $Q_{\mathrm{P}}$ also follows from the simple observation that the solution $\mathrm{I}^{\star}$ lies on a continuous path of configurations (NCL) connected to the vacuum configuration $W_{\text {vac }}=0$, which is topologically trivial $Q_{\mathrm{P}}(0)=0$.

\section{Solution}

The ansatz (8-10) is self-consistent, which means that the field equations (from $\delta A / \delta W=$ $\delta A / \delta \Phi=0$ ) reduce to 16 equations, which are precisely equal to those obtained from variations $\delta C_{i}$ and $\delta H_{j}$ of the ansatz action (31, 36). This agrees with the so-called principle of symmetric criticality [7], which states that in the quest of stationary points it suffices, under certain conditions, to consider variations that respect the symmetries of the ansatz (rotation and reflection symmetries in our case).

An analytic solution of the resulting non-linear partial differential equations for the functions $f_{i}(\rho, z, \tau)$ and $h_{j}(\rho, z, \tau)$ seems to be impossible and even an accurate numerical evaluation is a major enterprise, which we have to postpone for the moment. Still, it is possible to argue that there does exist a non-trivial solution, i. e. a solution different from the vacuum $\left(W=0,|\Phi|^{2}=\frac{1}{2} v^{2}\right)$ in whatever complicated gauge. The crucial observation is that the boundary conditions and symmetries of the ansatz imply the existence of at least one point where the total Higgs field vanishes $\Phi=0$, which is a gauge invariant statement.

Consider then the $H_{3}=0$ surface $\Sigma$ as it comes in from infinity (far out $\Sigma$ is, according to (13), given by the equation $z^{2}+\tau^{2}=\rho^{2}$ ). The simplest possibility is that of a single, connected surface as shown in Fig. 1a. The ansatz (9) gives, in that case, at least one point on the $\tau$-axis with all coefficient functions $H_{j}$ vanishing, i. e. $\Phi=0$ there. Alternatively, the $H_{3}=0$ surface could "pinch off", as shown in Fig. 1b, in which case 


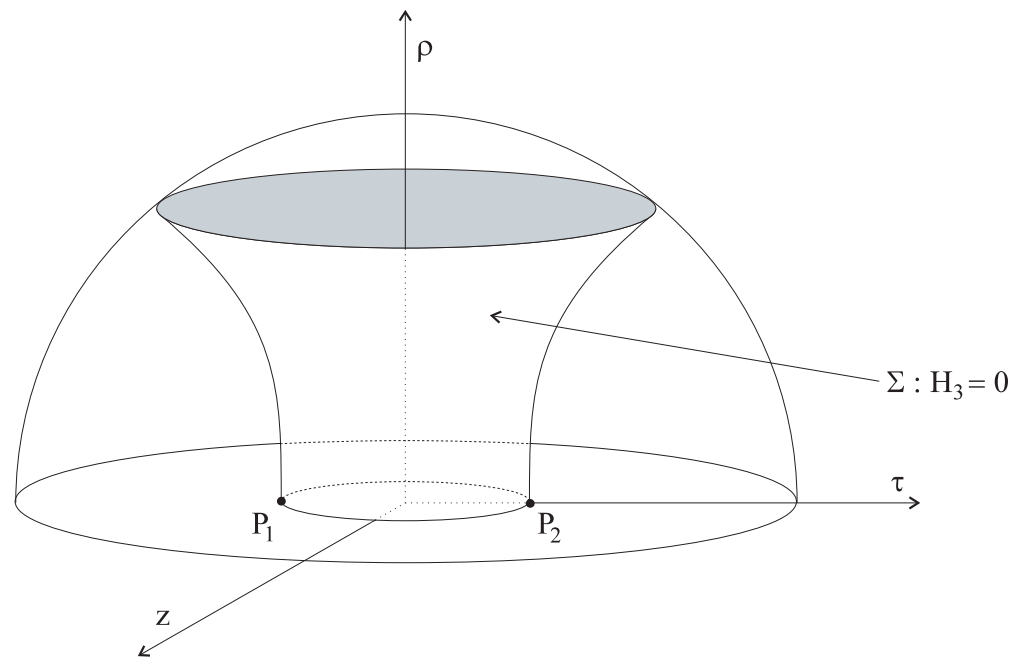

(a)

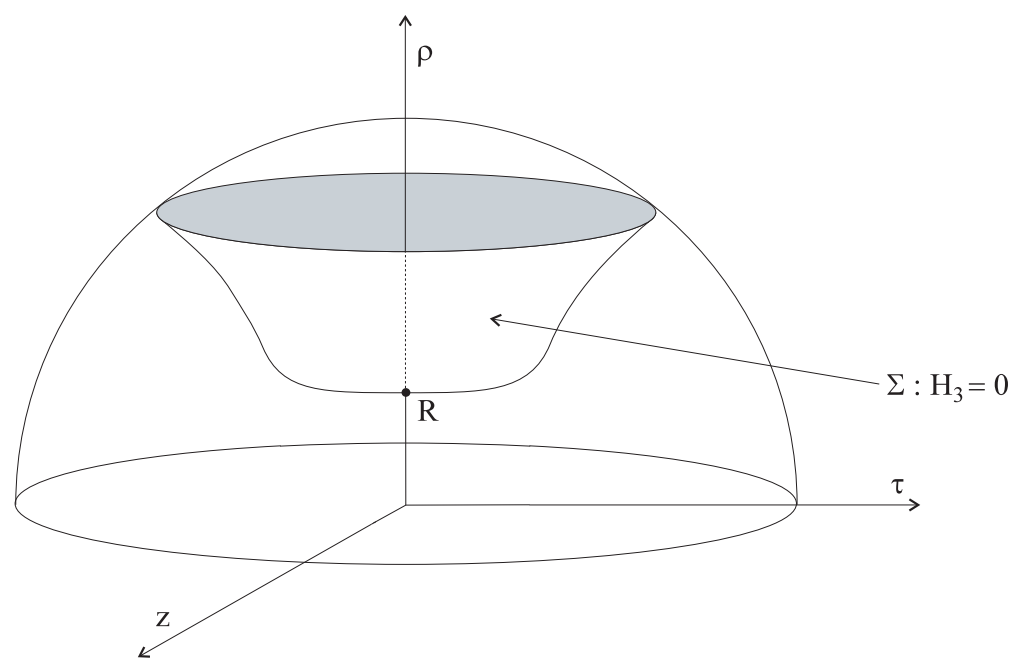

(b)

Figure 1: Sketch of the $H_{3}=0$ surface $\Sigma$ as it propagates in from the sphere at infinity. A priori there are two possibilities : (a) a single connected surface embedded in $\mathbb{R}^{4}$, with Higgs field vanishing at, generically, two points $P_{1}$ and $P_{2}$; (b) a "disconnected" surface in $\mathbb{R}^{4}$, with Higgs field vanishing at $R$, which corresponds to a ring of zeros in $\mathbb{R}^{4}$. 
the ansatz $(9,27)$ gives a ring of zeros for the Higgs field. Whichever possibility is realized is for the field equations to decide. The same two basic possibilities occur for the static sphaleron $\mathrm{S}^{\star}$, where the numerical solution of the field equations appears to indicate the first alternative [4]. Hence, one expects also $\mathrm{I}^{\star}$ to resemble a di-atomic molecule, albeit on a scale of $M_{\mathrm{W}}^{-1} \sim 10^{-8} \AA$.

\section{Discussion}

In this paper we have presented a selfconsistent ansatz for a new constrained instanton $\mathrm{I}^{\star}$ in $S U(2)$ Yang-Mills-Higgs theory. Further work on the numerical solution of the reduced field equations remains to be done, but we can already make some general remarks, see also [1, 네.

The constrained instanton $I^{\star}$ may be thought of as locating the top of the action barrier for the global $S U(2)$ anomaly [8]. The electroweak standard model has, of course, no global $S U(2)$ anomaly, the total number of left-handed fermion doublets being even. Still, the barrier structure in configuration space and the corresponding constrained instanton solution remain.

A more direct application of $I^{\star}$ to the electroweak interactions may be the asymptotics of Feynman perturbation theory. From the outset it is important to realize [9] that the electroweak standard model has only been established in (low order) perturbation theory. High-order contributions $c_{n} g^{2 n}$ to an arbitrary physical observable may be estimated [5] by saddle-point approximation of the euclidean path integral. It appears that $\mathrm{I}^{\star}$ provides the relevant saddle-point. In particular, the $\mathrm{I}^{\star}$ negative mode (which projects onto the non-contractible loop) plays a important role. Assuming there to be a single negative mode and assuming the integral over the collective coordinate $\varrho$ to be dominated by the value $\varrho=0$, the expected asymptotic behavior is

$$
c_{n} g^{2 n} \sim \frac{n !}{\left(A_{\mathrm{YMH}}^{\star}(0)\right)^{n}} \sim \frac{n !}{\left(16 \pi^{2}\right)^{n}} g^{2 n},
$$

where for the instanton action $A_{\mathrm{YMH}}^{\star}(\varrho)=16 \pi^{2} / g^{2}+\mathrm{O}\left(\varrho^{2} v^{2}\right)$ is used [1]. If this is indeed the asymptotic behaviour of standard electroweak perturbation theory, then the series is not even Borel summable. Physics considerations (such as causality and unitarity) should tell us how to make sense of perturbation theory or, more generally, how to define electroweak field theory non-perturbatively.

\section{Appendix A}

Here, we review the non-contractible loop (NCL) given in [1] and put it in the appropriate form for the construction of the ansatz of Section 3. 
The basic structure of this loop of 4-dimensional configurations is to first create and separate (to a distance $d$ ) an BPST-like instanton-anti-instanton pair, then make a complete relative isospin rotation, and finally collapse and annihilate the pair. For the present purpose we only need the isospin rotation part of the NCL with loop-parameter $\omega \in[-\pi / 2,+\pi / 2]$, whereas the whole NCL has $\omega$ running from $-3 \pi / 2$ to $+3 \pi / 2$.

With the same notation as in Section 3 and defining the radial coordinate $r^{2} \equiv \rho^{2}+z^{2}$, the configurations of the NCL are for $\omega \in[-\pi / 2,+\pi / 2]$

$$
\begin{aligned}
W_{\mu} & =-f \partial_{\mu} U U^{-1} \\
\Phi & =\frac{v}{\sqrt{2}} h U\left(\begin{array}{l}
0 \\
1
\end{array}\right),
\end{aligned}
$$

with the $S U(2)$ matrix

$$
U=i \sigma_{3} e^{(\omega+\pi / 2) i \sigma_{3}}\left(\hat{x}_{-} \cdot \sigma\right) e^{-(\omega+\pi / 2) i \sigma_{3}}\left(\hat{x}_{+} \cdot \sigma\right)^{\dagger}
$$

and definitions

$$
\begin{aligned}
\hat{x}_{ \pm}^{\mu} & \equiv x_{ \pm}^{\mu} /\left|x_{ \pm}\right| \\
x_{ \pm}^{\mu} & \equiv\left(x^{1}, x^{2}, x^{3}, x^{4} \pm d / 2\right) \\
\sigma^{\mu} & \equiv\left(i \sigma^{1}, i \sigma^{2}, i \sigma^{3}, \mathbb{1}_{2}\right) .
\end{aligned}
$$

The axial functions $f=f(r, \tau)$ and $h=h(r, \tau)$ have boundary conditions网

$$
\begin{gathered}
f(0, \pm d / 2)=h(0, \pm d / 2)=0 \\
\lim _{|x| \rightarrow \infty} f=\lim _{|x| \rightarrow \infty} h=1 .
\end{gathered}
$$

This completes our review of the crucial part of the NCL, which is based on the topologically non-trivial loop of mappings $U(\omega)$ of the sphere at infinity $S_{\infty}^{3}$ into the group manifold $S U(2)=S^{3}$.

The NCL configurations (A.1) are axially symmetric and in the notation of (8) the corresponding coefficient functions $C_{i}$ and $H_{j}$ are given by

$$
\begin{aligned}
C_{1}= & \frac{\rho f}{x_{+}^{2} x_{-}^{2}}\left\{\left((2 \tau-d)\left(\tau^{2}-\frac{d^{2}}{4}+z^{2}\right)-2 z^{2} d\right)-2 z x_{+}^{2} \sin 2 \omega\right. \\
& \left.+(2 \tau-d) x_{+}^{2} \cos 2 \omega-2 z \rho^{2} \sin 4 \omega+\rho^{2}(2 \tau+d) \cos 4 \omega\right\}
\end{aligned}
$$

${ }^{*}$ the functions $g_{ \pm}$of [1] are set to unity and a global gauge transformation (28) with $\Gamma=i \sigma_{3}$ has been performed.

** the additional conditions $f(r,-\tau)=f(r, \tau)$ and $h(r,-\tau)=h(r, \tau)$ of [1] are, strictly speaking, not essential. 


$$
\begin{aligned}
& C_{2}=-\frac{\rho f}{x_{+}^{2} x_{-}^{2}}\left\{2 z\left(\tau d+\tau^{2}-\frac{3}{4} d^{2}+z^{2}\right)+(2 \tau-d) x_{+}^{2} \sin 2 \omega\right. \\
& \left.+2 z x_{+}^{2} \cos 2 \omega+\rho^{2}(d+2 \tau) \sin 4 \omega+2 z \rho^{2} \cos 4 \omega\right\} \\
& C_{3}=\frac{\rho f}{x_{+}^{2} x_{-}^{2}}\left\{2 z\left(\tau d+\tau^{2}-\frac{3}{4} d^{2}+z^{2}\right)-(2 \tau-d)\left(2 \rho^{2}-x_{+}^{2}\right) \sin 2 \omega\right. \\
& \left.-2 z\left(2 \rho^{2}-x_{+}^{2}\right) \cos 2 \omega-\rho^{2}(2 \tau+d) \sin 4 \omega-2 z \rho^{2} \cos 4 \omega\right\} \\
& C_{4}=\frac{\rho f}{x_{+}^{2} x_{-}^{2}}\left\{\left((2 \tau-d)\left(\tau^{2}-\frac{d^{2}}{4}+z^{2}\right)-2 z^{2} d\right)+2 z\left(2 \rho^{2}-x_{+}^{2}\right) \sin 2 \omega\right. \\
& \left.-(2 \tau-d)\left(2 \rho^{2}-x_{+}^{2}\right) \cos 2 \omega+2 z \rho^{2} \sin 4 \omega-\rho^{2}(2 \tau+d) \cos 4 \omega\right\} \\
& C_{5}=\frac{4 \rho^{2} f}{x_{+}^{2} x_{-}^{2}}\left\{\left(\tau^{2}+z^{2}+\frac{d^{2}}{4}\right)+d z \sin 2 \omega+\left(\tau^{2}+z^{2}-\frac{d^{2}}{4}\right) \cos 2 \omega\right\} \\
& C_{6}=\frac{4 \rho^{2} f}{x_{+}^{2} x_{-}^{2}}\left\{\left(\frac{d^{2}}{4}-z^{2}-\tau^{2}\right) \sin 2 \omega+d z \cos 2 \omega\right\} \\
& C_{7}=-2 \frac{\rho z f}{x_{+}^{2} x_{-}^{2}}\left\{z(2 \tau-d)-\left(\tau d+z^{2}+\rho^{2}-\tau^{2}+\frac{3}{4} d^{2}\right) \sin 2 \omega\right. \\
& \left.+z(d+2 \tau) \cos 2 \omega-\rho^{2} \sin 4 \omega\right\} \\
& C_{8}=2 \frac{\rho z f}{x_{+}^{2} x_{-}^{2}}\left\{\left(\tau^{2}-z^{2}-\tau d+\frac{d^{2}}{4}\right)-\left(\tau d+z^{2}+\rho^{2}-\tau^{2}+\frac{3}{4} d^{2}\right) \cos 2 \omega\right. \\
& \left.-z(2 \tau+d) \sin 2 \omega-\rho^{2} \cos 4 \omega\right\} \\
& C_{9}=2 \frac{z f}{x_{+}^{2} x_{-}^{2}}\left\{\left(2 \rho^{2} \tau+\tau^{2} d-z^{2} d-\frac{d^{3}}{4}\right)+2 z \rho^{2} \sin 2 \omega+\rho^{2}(2 \tau-d) \cos 2 \omega\right\} \\
& C_{10}=2 \frac{\rho \tau f}{x_{+}^{2} x_{-}^{2}}\left\{\left(z^{2}-\tau^{2}+\tau d-\frac{d^{2}}{4}\right)-\left(\rho^{2}+\tau d-z^{2}+\frac{d^{2}}{4}+\tau^{2}\right) \cos 2 \omega\right. \\
& \left.+z(2 \tau-d) \sin 2 \omega-\rho^{2} \cos 4 \omega\right\} \\
& C_{11}=-2 \frac{\rho \tau f}{x_{+}^{2} x_{-}^{2}}\left\{z(2 \tau-d)+\left(\rho^{2}+\tau d-z^{2}+\frac{d^{2}}{4}+\tau^{2}\right) \sin 2 \omega\right. \\
& \left.+z(2 \tau-d) \cos 2 \omega+\rho^{2} \sin 4 \omega\right\}
\end{aligned}
$$




$$
\begin{aligned}
& C_{12}=-4 \frac{f \tau}{x_{+}^{2} x_{-}^{2}}\left\{z\left(\tau d+\rho^{2}\right)+\rho^{2}\left(\frac{d}{2}-\tau\right) \sin 2 \omega+\rho^{2} z \cos 2 \omega\right\} \\
& H_{0}=\frac{h}{x_{+} x_{-}}\left\{-z d+\rho^{2} \sin 2 \omega\right\} \\
& H_{1}=-\frac{h}{x_{+} x_{-}} \rho\left\{z+\left(\tau+\frac{d}{2}\right) \sin 2 \omega+z \cos 2 \omega\right\} \\
& H_{2}=-\frac{h}{x_{+} x_{-}} \rho\left\{\left(\tau-\frac{d}{2}\right)-z \sin 2 \omega+\left(\tau+\frac{d}{2}\right) \cos 2 \omega\right\} \\
& H_{3}=\frac{h}{x_{+} x_{-}}\left\{\left(\frac{d^{2}}{4}-z^{2}-\tau^{2}\right)+\rho^{2} \cos 2 \omega\right\} .
\end{aligned}
$$

Only for $\omega=0$ (and $\omega= \pm \pi / 2$ ) do we have the discrete symmetries described in Section 4.2. In $H_{0}$, for example, there is a term proportional to $\rho^{2} \sin 2 \omega$ that breaks the discrete symmetry (22), whereas the other term proportional to $z d$ respects it.

The ansatz of Section 3 then is a generalization of the configuration (A.4) at the top $(\omega=0)$ of this particular NCL, keeping the axial and discrete symmetries present and taking over the boundary conditions at infinity. In fact, the ansatz (8, 9) has a priori 16 independent functions $f_{i}(\rho, z, \tau)$ and $h_{j}(\rho, z, \tau)$, whereas the maximal configuration of the NCL (A.1) has only two, namely $f(r, \tau)$ and $h(r, \tau)$. This illustrates the degree of generalization required to obtain an exact solution of the field equations.

\section{Appendix B}

Here, we list the transformation properties of the coefficient functions $C_{i}, H_{j}$ of the ansatz (8) under the residual gauge symmetries (28, 29).

The transformations of the gauge field coefficients functions $C_{i}(i=1, \ldots, 12)$ are the 
following:

$$
\begin{aligned}
&\left(\begin{array}{c}
C_{1} \\
C_{2} \\
C_{6}
\end{array}\right) \rightarrow\left(\begin{array}{c}
T_{\rho 1} \\
T_{\rho 2} \\
T_{\rho 3}
\end{array}\right)+R\left(\begin{array}{c}
C_{1} \\
C_{2} \\
C_{6}
\end{array}\right) \\
&\left(\begin{array}{c}
C_{3} \\
C_{4} \\
1-C_{5}
\end{array}\right) \rightarrow R\left(\begin{array}{c}
C_{3} \\
C_{4} \\
1-C_{5}
\end{array}\right) \\
&\left(\begin{array}{c}
C_{7} \\
-C_{8} \\
C_{9}
\end{array}\right) \rightarrow\left(\begin{array}{c}
T_{z 1} \\
T_{z 2} \\
T_{z 3}
\end{array}\right)+R\left(\begin{array}{c}
C_{7} \\
-C_{8} \\
C_{9}
\end{array}\right) \\
&\left(\begin{array}{c}
C_{10} \\
-C_{11} \\
C_{12}
\end{array}\right) \rightarrow\left(\begin{array}{c}
T_{\tau 1} \\
T_{\tau 2} \\
T_{13} \\
-C_{11} \\
C_{12}
\end{array}\right)+R
\end{aligned}
$$

with the $S O(3)$ matrix

$$
R \equiv\left(\begin{array}{ccc}
R_{11} & R_{12} & R_{13} \\
R_{21} & R_{22} & R_{23} \\
R_{31} & R_{32} & R_{33}
\end{array}\right)
$$

defined in terms of

$$
\begin{aligned}
R_{c a} & \equiv \frac{1}{\Omega^{2}}\left[\omega_{c} \omega_{a}(1-\cos \Omega)-\epsilon_{c a b} \omega_{b} \Omega \sin \Omega+\delta_{c a} \Omega^{2} \cos \Omega\right] \\
T_{\bar{\alpha} c} & \equiv \frac{1}{\Omega^{2}}\left(\bar{\alpha} \partial_{\bar{\alpha}} \omega_{a}\right)\left[\omega_{c} \omega_{a}\left(\frac{\sin \Omega}{\Omega}-1\right)+\epsilon_{c a b} \omega_{b}(1-\cos \Omega)-\delta_{c a} \Omega \sin \Omega\right] \\
\Omega^{2} & \equiv \omega_{1}^{2}+\omega_{2}^{2}+\omega_{3}^{2}
\end{aligned}
$$

where $\bar{\alpha}$ stands for the variables $\rho, z, \tau$, and the indices $a, b, c$ run over the values $1,2,3$ (as always, there is the summation convention of repeated indices).

The transformations of the Higgs field coefficient functions $H_{j}(j=0, \ldots, 3)$ are the following :

$$
\left(\begin{array}{c}
H_{0} \\
H_{1} \\
H_{2} \\
H_{3}
\end{array}\right) \rightarrow R_{\mathrm{H}}\left(\begin{array}{c}
H_{0} \\
H_{1} \\
H_{2} \\
H_{3}
\end{array}\right)
$$

with the $S O(4)$ matrix

$$
R_{\mathrm{H}}=\cos \left(\frac{\Omega}{2}\right) \mathbb{1}_{4}+\sin \left(\frac{\Omega}{2}\right) \frac{\vec{\omega} \cdot \vec{S}}{\Omega}
$$


defined in terms of the 4-dimensional identity matrix $\mathbb{1}_{4}$ and

$$
S_{1} \equiv\left(\begin{array}{cc}
-i \sigma_{2} & 0 \\
0 & -i \sigma_{2}
\end{array}\right) \quad S_{2} \equiv\left(\begin{array}{cc}
0 & -\sigma_{3} \\
\sigma_{3} & 0
\end{array}\right) \quad S_{3} \equiv\left(\begin{array}{cc}
0 & -\sigma_{1} \\
\sigma_{1} & 0
\end{array}\right) \text {. }
$$

\section{Appendix $\mathrm{C}$}

Here, we give the ansatz actiondensities $a_{\mathrm{WKIN}}, a_{\mathrm{HKIN}}, a_{\mathrm{HPOT}}$ and Pontryagin density $q_{\mathrm{P}}$ in a compact, transparent notation.

First, define the 3-dimensional coordinates

$$
\left(y_{1}, y_{2}, y_{3}\right) \equiv(\rho, z, \tau)
$$

Second, introduce the following "isovectors" given in terms of the coefficient functions $C_{i}$ of the ansatz (8):

$$
\vec{k}_{1} \equiv\left(\begin{array}{c}
C_{1} \\
C_{2} \\
C_{6}
\end{array}\right) \quad \vec{k}_{2} \equiv\left(\begin{array}{c}
C_{7} \\
-C_{8} \\
C_{9}
\end{array}\right) \quad \vec{k}_{3} \equiv\left(\begin{array}{c}
C_{10} \\
-C_{11} \\
C_{12}
\end{array}\right) \quad \vec{k}_{4} \equiv\left(\begin{array}{c}
C_{3} \\
C_{4} \\
1-C_{5}
\end{array}\right),
$$

which are motivated by the transformation properties (B.1) found in Appendix B. Third, define the "field strengths" and "covariant derivatives" as

$$
\begin{aligned}
\vec{K}_{\alpha \beta} & \equiv \frac{\partial}{\partial y_{\alpha}}\left(\frac{\vec{k}_{\beta}}{y_{\beta}}\right)-\frac{\partial}{\partial y_{\beta}}\left(\frac{\vec{k}_{\alpha}}{y_{\alpha}}\right)+\frac{\vec{k}_{\alpha}}{y_{\alpha}} \times \frac{\vec{k}_{\beta}}{y_{\beta}}, \\
D_{\alpha} \vec{k}_{4} & \equiv \frac{\partial}{\partial y_{\alpha}} \vec{k}_{4}+\frac{\vec{k}_{\alpha}}{y_{\alpha}} \times \vec{k}_{4},
\end{aligned}
$$

where the indices $\alpha, \beta$ take the values 1, 2, 3. With these definitions the Yang-Mills actiondensity (33) and Pontryagin density (37) become simply

$$
\begin{aligned}
a_{\mathrm{WKIN}} & =\frac{1}{2 g^{2}}\left\{\frac{1}{2} \sum_{\alpha, \beta=1}^{3}\left|\vec{K}_{\alpha \beta}\right|^{2}+\sum_{\alpha=1}^{3}\left|\frac{1}{\rho} D_{\alpha} \vec{k}_{4}\right|^{2}\right\} \\
q_{\mathrm{P}} & =\frac{1}{2 \rho} \sum_{\alpha, \beta, \gamma=1}^{3} \epsilon_{\alpha \beta \gamma} \frac{\partial}{\partial y_{\gamma}}\left(\vec{k}_{4} \cdot \vec{K}_{\alpha \beta}\right) .
\end{aligned}
$$

For the Higgs actiondensity it turns out to be useful to introduce "4-vectors", together with an implicit euclidean metric of positive signature. First, define the following 4 -vector in terms of the coefficient functions $H_{j}$ of the ansatz (8) :

$$
H^{\mu} \equiv\left(\begin{array}{c}
H_{0} \\
H_{1} \\
H_{2} \\
H_{3}
\end{array}\right)
$$


Second, introduce the "covariant derivatives"

$$
\mathcal{D}_{\alpha} \equiv \frac{\partial}{\partial y_{\alpha}} \mathbb{1}_{4}+\frac{\vec{k}_{\alpha}}{y_{\alpha}} \cdot \frac{\vec{S}}{2},
$$

with $\alpha=1,2,3$, and the $4 \times 4$ matrices

$$
S_{1} \equiv\left(\begin{array}{cc}
-i \sigma_{2} & 0 \\
0 & -i \sigma_{2}
\end{array}\right) \quad S_{2} \equiv\left(\begin{array}{cc}
0 & -\sigma_{3} \\
\sigma_{3} & 0
\end{array}\right) \quad S_{3} \equiv\left(\begin{array}{cc}
0 & -\sigma_{1} \\
\sigma_{1} & 0
\end{array}\right),
$$

which appeared already in the transformations (B.2) of Appendix B. Third, define the matrix

$$
K_{4} \equiv \vec{k}_{4} \cdot \vec{S}+T_{3},
$$

where

$$
T_{3} \equiv\left(\begin{array}{cc}
0 & i \sigma_{2} \\
i \sigma_{2} & 0
\end{array}\right)
$$

commutes with all $S_{a}$. With these definitions the Higgs actiondensities (34) and (35) become

$$
\begin{aligned}
& a_{\text {HКIN }}=\frac{v^{2}}{2}\left\{\sum_{\alpha=1}^{3}\left(\mathcal{D}_{\alpha}^{\mu \nu} H^{\nu}\right)^{2}+\left(\frac{1}{2 \rho} K_{4}^{\mu \nu} H^{\nu}\right)^{2}\right\} \\
& a_{\text {Hрот }}=\lambda \frac{v^{4}}{4}\left(H^{\mu} H^{\mu}-1\right)^{2},
\end{aligned}
$$

where the last term in $a_{\text {HKIN }}$ mixes the two types of "scalars" $\left(\vec{k}_{4}\right.$ and $\left.H^{\mu}\right)$ of the effective $S O(3)$ Yang-Mills-Higgs theory found.

\section{References}

[1] F. Klinkhamer, Nucl. Phys. B407 (1993) 88

[2] A. Belavin, A. Polyakov, A. Schwartz and Yu. Tyupkin, Phys. Lett. 59B (1975) 85

[3] M. Atiyah, V. Drinfeld, N. Hitchin and Yu. Manin, Phys. Lett. 65A (1978) 185

[4] F. Klinkhamer, Nucl. Phys. B410 (1993) 343

[5] L. Lipatov, Sov. Phys. JETP 45 (1977) 216

[6] I. Affleck, Nucl. Phys. B191 (1981) 429

[7] R. Palais, Comm. Math. Phys. 69 (1979) 19

[8] E. Witten, Phys. Lett. 117 B (1982) 324

[9] M. Veltman, Diagrammatica - The Path to Feynman Rules, Cambridge UP (1994) 